\title{
Antimicrobial resistance surveillance in the South African private sector report for 2016
}

\author{
Olga Perovic $^{a, b *}$, Husna Ismail ${ }^{a}$, Erika Van Schalkwyk ${ }^{a}$, Warren Lowman ${ }^{b, c, d}$, Elizabeth Prentice ${ }^{e}$, Marthinus Senekal ${ }^{f}$ and \\ Chetna N Govind ${ }^{\text {g,h }}$
}

${ }^{a}$ Centre for Healthcare-Associated Infections (HAls), Antimicrobial Resistance (AMR) and Mycoses, National Institute for Communicable Diseases, a division in the National Health Laboratory Service, Johannesburg, South Africa

${ }^{b}$ Department of Clinical Microbiology and Infectious Diseases, School of Pathology, University of Witwatersrand, Johannesburg, South Africa 'Department of Clinical Microbiology, Vermaak and Partners/Pathcare Pathologists, Pretoria, South Africa

${ }^{d}$ Wits Donald Gordon Medical Centre, Johannesburg, South Africa

${ }^{e}$ Ampath Microbiology National Reference Laboratory, Centurion, South Africa

${ }^{f}$ Pathcare Pathology Group Reference Laboratory, Goodwood, Western Cape, South Africa

${ }^{g}$ Department of Clinical Microbiology, Lancet Laboratories, Durban, South Africa

${ }^{h}$ College of Health Sciences, University of KwaZulu-Natal, Durban, South Africa

*Corresponding author, email: olgap@nicd.ac.za

Aim: The relevance of surveillance for antimicrobial resistance is increasingly recognised in the light of a global action plan to combat resistance. This report presents antimicrobial susceptibility testing on ESKAPE pathogens from private sector laboratories in South Africa for 2016

Methods: Antimicrobial susceptibility testing (AST) performed on ESKAPE organisms (Enterococcus faecium, Enterococcus faecalis, Staphylococcus aureus, Klebsiella pneumoniae, Acinetobacter baumannii, Pseudomonas aeruginosa, Enterobacter cloacae and Escherichia coli) isolated from blood cultures at four private pathology laboratories in 2016 were analysed. Analysis and reporting of data were done via a uniform platform created by the NICD for national AST data.

Results: AST were reported on 9029 ESKAPE organisms including 58\% Enterobacteriaceae, 28\% Gram-positive bacteria and 14\% Gram-negative bacteria and drug-bug combination was performed following the Global Antimicrobial Surveillance System (GLASS) guidelines by the World Health Organization.

Conclusions: The most important resistance to address is a high level of ESBL in Enterobacteriaceae, which necessitates the use of carbapenems for treatment. Resistance to carbapenems is recorded in this report but not confirmation of genes by genotypic methods. During this period, no increase in vancomycin-resistant Enterococci was observed.

Keywords: antimicrobial resistance, surveillance, ESKAPE organisms

\section{Introduction}

Over the last 20 years, antimicrobial resistance (AMR) has reached a pandemic level. ${ }^{1}$ According to estimates by the US Centres for Disease Control and Prevention, each year more than two million people are infected with antimicrobial-resistant microorganisms of whom 23000 die due to these infections. ${ }^{1}$ The Global Report on Surveillance by the World Health Organization indicated that AMR is on the increase in Africa. ${ }^{2}$ However, accurate and reliable data are limited and as a result the true extent of the problem is unknown. ${ }^{2}$ Here we report on a select group of bacteria that not only cause healthcare-associated infections, but also effectively 'escape' the effects of antimicrobial agents. This group of bacteria is known by the acronym, ESKAPE. ${ }^{3}$ The aim of this report was to describe the spectrum of ESKAPE pathogens (Enterococcus faecium, Enterococcus faecalis, Staphylococcus aureus, Klebsiella pneumoniae, Acinetobacter baumannii, Pseudomonas aeruginosa, Enterobacter cloacae and Escherichia coli) together with their antimicrobial susceptibility testing (AST) patterns identified from private-sector laboratories across South Africa.

\section{Methods}

Secondary data analysis was conducted on ESKAPE isolates identified from four accredited private sector pathology laboratories (Ampath, Lancet Laboratories, PathCare and Vermaak and Partners) located in all nine provinces in South Africa, from January to December 2016. The study population was all patients who had a blood culture submitted to a private laboratory. We could not distinguish hospital-associated infections from community-associated infections as admission data were not available at the time of analysis. Positive blood cultures with any one of the ESKAPE organisms isolated were included in the analysis. A 21-day de-duplication rule was applied to all positive blood cultures. AST and interpretation of results were performed according to Clinical and Laboratory Standards Institute (CLSI) 2016 guidelines. ${ }^{4}$ AST results were categorised based on categorical data, i.e. susceptible (S) and non-susceptible, including intermediate (I) and resistant (R). Reporting of susceptibility patterns for drug-bug combinations was performed based on the Global Antimicrobial Surveillance System (GLASS) manual. ${ }^{5}$ No patient demographic or clinical data were available. AST data for specified ESKAPE isolates were extracted from a secure web-based electronic platform created by the Surveillance Information Management Unit (SIMU) at the National Institute for Communicable Diseases (NICD). These data are available on the AMR dashboard from the NICD website accessible at http://www.nicd.ac.za. Absolute frequencies, percentages, bar charts and tables were used to describe the data. 


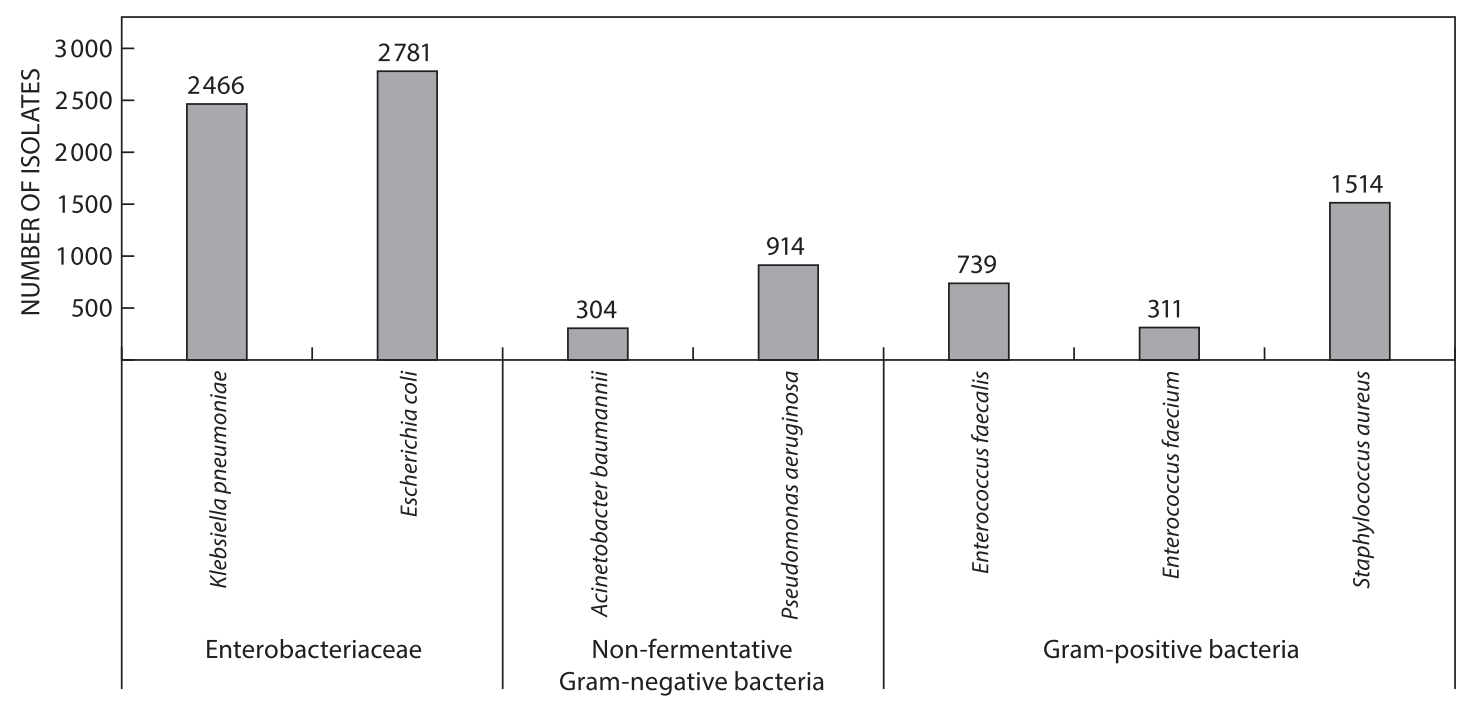

Figure 1: Number of ESKAPE isolates identified from blood cultures, January to December 2016.

\section{Results}

For the purpose of this report, ESKAPE pathogen profiles were categorised as Enterobacteriaceae (Klebsiella pneumoniae and Escherichia coli), non-fermentative Gram-negative bacteria (Acinetobacter baumannii and Pseudomonas aeruginosa) and Gram-positive bacteria (Enterococcus faecalis, Enterococcus faecium and Staphylococcus aureus). Of the 9029 blood cultures that grew ESKAPE organisms, 58\% (5 247) were Enterobacteriaceae, 28\% ( $n=2564)$ were Gram-positive bacteria and 14\% $(n=$ 1218) were non-fermentative Gram-negative bacteria. Of the seven ESKAPE organisms, Escherichia coli, Klebsiella pneumoniae and Staphylococcus aureus were the three organisms most commonly isolated from blood cultures (Figure 1).

\section{Enterobacteriaceae}

Klebsiella pneumoniae

Forty-three per cent and $20 \%$ of $K$. pneumoniae isolates were non-susceptible to the aminoglycosides, gentamicin and amikacin, respectively. Forty-one per cent and $25 \%$ of isolates were non-susceptible to the quinolones, ciprofloxacin and levofloxacin, respectively. Non-susceptibility to the third-generation cephalosporins, cefotaxime/ceftriaxone and ceftazidime, was from $55 \%$ to $57 \%$, while non-susceptibility to the fourth-generation cephalosporin, cefepime, was $56 \%$. Fifty-seven per cent of isolates were non-susceptible to the beta-lactam + beta-lactamase inhibitor, piperacillin/tazobactam. Less than $10 \%$ of isolates were non-susceptible to the carbapenems: imipenem, meropenem and doripenem, but $15 \%$ were non-susceptible to ertapenem (Table 1).

\section{Escherichia coli}

Fifteen per cent and $6 \%$ of $E$. coli isolates were non-susceptible to the aminoglycosides, gentamicin and amikacin, respectively. Thirty-one per cent and $25 \%$ of isolates were non-susceptible to the quinolones, ciprofloxacin and levofloxacin, respectively. Non-susceptibility to the third-generation cephalosporins, cefotaxime/ceftriaxone and ceftazidime, was from $18 \%$ to $19 \%$, while non-susceptibility to the fourth-generation cephalosporin, cefepime was $18 \%$. Twenty per cent of isolates were non-susceptible to the beta-lactam + beta-lactamase inhibitor, piperacillin/tazobactam. Less than $1 \%$ of isolates were non-susceptible to the carbapenems: imipenem, meropenem, ertapenem and doripenem, respectively (Table 1 ).

\section{Non-fermentative Gram-negative bacteria Acinetobacter baumannii}

Forty-seven per cent and $37 \%$ of $A$. baumannii isolates were nonsusceptible to the aminoglycosides, gentamicin and amikacin, respectively. Non-susceptibility to the carbapenems, imipenem and meropenem, were from $54 \%$ to $56 \%$, while non-susceptibility to doripenem was observed in $53 \%$ of the isolates. Susceptibility patterns for tetracycline and minocycline were not reported; however, $10 \%$ of isolates were non-susceptible to tigecycline (Table 2).

\section{Pseudomonas aeruginosa}

Less than $30 \%$ of $P$. aeruginosa isolates were non-susceptible to the third-generation cephalosporin, ceftazidime, and the fourthgeneration cephalosporin, cefepime. Non-susceptibility to the carbapenems, meropenem and imipenem, was $36 \%$ and $38 \%$, while non-susceptibility to doripenem was observed in $32 \%$ of the isolates. Thirty-six per cent of isolates were non-susceptible to the beta-lactam + beta-lactamase inhibitor, piperacillin/tazobactam (Table 2).

\section{Gram-positive bacteria}

\section{Enterococcus species}

Enterococcus faecalis. Twenty-five per cent of E. faecalis isolates were non-susceptible to penicillin/ampicillin. Less than $1 \%$ of isolates were non-susceptible to the glycopeptides, teicoplanin and vancomycin, respectively. Less than $1 \%$ of isolates were non-susceptible to the oxazolidinone, linezolid (Table 3).

Enterococcus faecium. A high proportion of E. faecium isolates (92\%) were non-susceptible to penicillin/ampicillin. Four per cent and $5 \%$ of isolates were non-susceptible to the glycopeptides, teicoplanin and vancomycin, respectively. Low levels of non-susceptibility were observed in $2 \%$ of isolates to the oxazolidinone, linezolid (Table 3).

Staphylococcus aureus. Twenty-six per cent of S. aureus isolates were non-susceptible to cloxacillin and could be classified as methicillin-resistant S. aureus (MRSA) (Table 4).

\section{Discussion}

Almost the same number of ESKAPE pathogens were isolated from blood cultures in public sector, 10952 compared with 
Table 1: Antimicrobial susceptibility patterns of Enterobacteriaceae isolated from blood cultures reported from four private groups in South Africa, January 1, 2016 to December 31, 2016

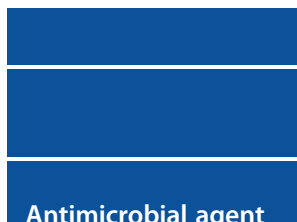

Amikacin

Amoxicillin-clavulanic acid

Ampicillin/amoxicillin

Cefepime

Cefotaxime/ceftriaxone

Ceftazidime

Ciprofloxacin

Cotrimoxazole

Doripenem

Ertapenem

Gentamicin

Imipenem

Levofloxacin

Meropenem

Piperacillin/

tazobactam

Abbreviations: number of isolates $(n)$, percentage (\%), not reported (-).

\begin{tabular}{|c|c|c|c|c|}
\hline \multicolumn{4}{|c|}{ Klebsiella pneumoniae complex } \\
\hline & \multicolumn{2}{|c|}{$\begin{array}{c}\text { Non- } \\
\text { susceptible }\end{array}$} & \multicolumn{2}{c|}{ Susceptible } \\
\hline $\begin{array}{c}\text { Number of isolates } \\
\text { tested }\end{array}$ & $n$ & $\%$ & $n$ & $\%$ \\
\hline 2444 & 480 & 19.6 & 1964 & 80.4 \\
\hline 2450 & 1475 & 60.2 & 975 & 39.8 \\
\hline
\end{tabular}

\begin{tabular}{|c|c|}
\hline \multicolumn{2}{|c}{ Esch } \\
\hline $\begin{array}{c}\text { Number of isolates } \\
\text { tested }\end{array}$ \\
\hline 2781
\end{tabular}

Escherichia coli

\begin{tabular}{|c|c|c|c|}
\hline \multicolumn{2}{|c|}{$\begin{array}{c}\text { Non- } \\
\text { susceptible }\end{array}$} & \multicolumn{2}{c|}{ Susceptible } \\
\hline$n$ & $\%$ & $n$ & $\%$ \\
\hline 183 & 6.6 & 2598 & 93.4 \\
\hline 835 & 30.0 & 1945 & 70.0 \\
\hline 1569 & 78.5 & 425 & 21.3 \\
\hline 495 & 17.8 & 2283 & 82.2 \\
\hline 524 & 18.9 & 2253 & 81.1 \\
\hline 393 & 18.3 & 1755 & 81.7 \\
\hline 619 & 31.0 & 1378 & 69.0 \\
\hline 1089 & 62.4 & 657 & 37.6 \\
\hline 5 & 0.2 & 2748 & 99.8 \\
\hline 10 & 0.4 & 2769 & 99.6 \\
\hline 411 & 14.8 & 2368 & 85.2 \\
\hline 5 & 0.2 & 2772 & 99.8 \\
\hline 199 & 25.1 & 593 & 74.9 \\
\hline 3 & 0.1 & 2777 & 99.9 \\
\hline 562 & 20.3 & 2212 & 79.7 \\
\hline & & & \\
\hline
\end{tabular}

Colistin was not reported as no reference method was applied at private-sector laboratories.

9029 from private laboratories (unpublished data) in 2016. In the private sector $56 \%$ of $K$. pneumoniae isolates $(n=2$ 466) were resistant to third and fourth generation cephalosporins compared with $65 \%(n=2783)$ in the public sector (unpublished data). Of the total number of $E$. coli isolates $(n=2$ 781), 18\% showed non-susceptibility to third and fourth generation cephalosporins in the private sector compared with $28 \%$ of $E$. coli isolates $(n=1850)$ in the public sector, while $30 \%$ were non-susceptible to ciprofloxacin in both sectors.
Regarding non-fermentative Gram-negative bacteria, 56\% of A. baumannii isolates $(n=304)$ were non-susceptible to carbapenems in the private sector while $80 \%(n=2318)$ in the public sector.

$P$. aeruginosa isolates showed similar susceptibility patterns in both public and private sectors.

Gram-positive organisms showed very stable susceptibility patterns and no vancomycin-resistant Enterococci outbreaks were

Table 2: Antimicrobial susceptibility patterns of non-fermenters isolated from blood cultures reported from four private groups in South Africa, January 1, 2016 to December 31, 2016

\begin{tabular}{|c|c|c|c|c|c|c|c|c|c|c|}
\hline \multirow[b]{3}{*}{ Antimicrobial agent } & \multicolumn{5}{|c|}{ Acinetobacter baumannii } & \multicolumn{5}{|c|}{ Pseudomonas aeruginosa } \\
\hline & \multirow[b]{2}{*}{$\begin{array}{c}\text { Number of isolates } \\
\text { tested }\end{array}$} & \multicolumn{2}{|c|}{$\begin{array}{c}\text { Non- } \\
\text { susceptible }\end{array}$} & \multicolumn{2}{|c|}{ Susceptible } & \multirow[b]{2}{*}{$\begin{array}{c}\text { Number of isolates } \\
\text { tested }\end{array}$} & \multicolumn{2}{|c|}{$\begin{array}{c}\text { Non- } \\
\text { susceptible }\end{array}$} & \multicolumn{2}{|c|}{ Susceptible } \\
\hline & & $n$ & $\%$ & $n$ & $\%$ & & $n$ & $\%$ & $n$ & $\%$ \\
\hline Amikacin & 288 & 106 & 36.8 & 182 & 63.2 & - & - & - & - & - \\
\hline Cefepime & - & - & - & - & - & 908 & 256 & 28.2 & 652 & 71.8 \\
\hline Ceftazidime & - & - & - & - & - & 892 & 235 & 26.3 & 657 & 73.7 \\
\hline Doripenem & 257 & 137 & 53.3 & 120 & 46.7 & 883 & 282 & 31.9 & 601 & 68.1 \\
\hline Gentamicin & 303 & 142 & 46.9 & 161 & 53.1 & - & - & - & - & - \\
\hline Imipenem & 304 & 165 & 54.3 & 139 & 45.7 & 911 & 344 & 37.8 & 567 & 62.2 \\
\hline Meropenem & 304 & 171 & 56.3 & 133 & 43.8 & 912 & 324 & 35.5 & 588 & 64.5 \\
\hline $\begin{array}{l}\text { Piperacillin/ } \\
\text { tazobactam }\end{array}$ & & & & & & 902 & 320 & 35.5 & 582 & 64.5 \\
\hline Tigecycline & 212 & 22 & 10.4 & 190 & 89.6 & - & - & - & - & - \\
\hline
\end{tabular}

Abbreviations: number of isolates ( $n$ ), percentage (\%), not reported (-).

Colistin was not reported as no reference method was applied at private-sector laboratories. 
Table 3: Antimicrobial susceptibility patterns of enterococci species isolated from blood cultures reported from four private groups in South Africa, January 1, 2016 to December 31, 2016

\begin{tabular}{|c|c|c|c|c|c|c|c|c|c|c|}
\hline & \multicolumn{5}{|c|}{ Enterococcus faecalis } & \multicolumn{5}{|c|}{ Enterococcus faecium } \\
\hline & \multirow[b]{2}{*}{$\begin{array}{c}\text { Number of isolates } \\
\text { tested }\end{array}$} & \multicolumn{2}{|c|}{$\begin{array}{l}\text { Non- } \\
\text { susceptible }\end{array}$} & \multicolumn{2}{|c|}{ Susceptible } & \multirow[b]{2}{*}{$\begin{array}{c}\text { Number of isolates } \\
\text { tested }\end{array}$} & \multicolumn{2}{|c|}{$\begin{array}{c}\text { Non- } \\
\text { susceptible }\end{array}$} & \multicolumn{2}{|c|}{ Susceptible } \\
\hline $\begin{array}{l}\text { Antimicrobial } \\
\text { agent }\end{array}$ & & $n$ & $\%$ & $n$ & $\%$ & & $n$ & $\%$ & $n$ & $\%$ \\
\hline Daptomycin & 168 & $\mathrm{~N} / \mathrm{A}$ & $\mathrm{N} / \mathrm{A}$ & 168 & 100.0 & 65 & N/A & N/A & 63 & 96.9 \\
\hline Linezolid & 511 & 3 & 0.6 & 508 & 99.4 & 215 & 5 & 2.3 & 210 & 97.7 \\
\hline Penicillin/ampicillin & 88 & 22 & 25.0 & 66 & 75.0 & 38 & 35 & 92.1 & 3 & 7.9 \\
\hline Teicoplanin & 695 & 3 & 0.4 & 692 & 99.6 & 295 & 12 & 4.1 & 283 & 95.9 \\
\hline Vancomycin & 726 & 2 & 0.3 & 724 & 99.7 & 309 & 14 & 4.5 & 295 & 95.5 \\
\hline
\end{tabular}

Abbreviations: number of isolates (n), percentage (\%).

Table 4: Antimicrobial susceptibility patterns of Staphylococcus aureus isolated from blood cultures reported from four private groups in South Africa, January 1, 2016 to December 31, 2016

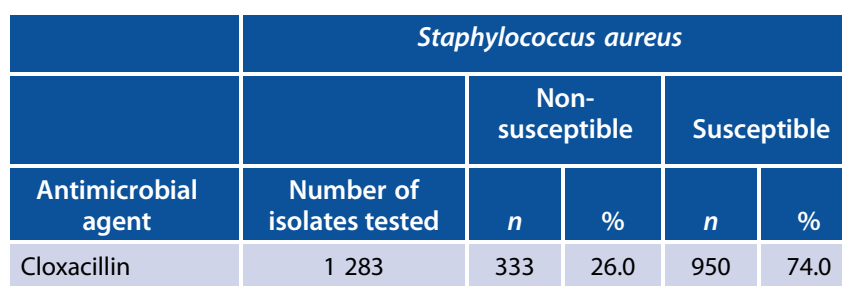

Abbreviations: number of isolates (n), percentage (\%).

noted during this period. MRSA was identified in $30 \%$ of S. aureus isolates ( $n=2338$ ) from the public sector and $26 \%$ isolates $(n=1283)$ in the private sector.

\section{Limitations}

In this report, not all private laboratories were included (only the four largest private groups). Another limitation is the different practices employed by private laboratories, which may influence specimen submission practices and the testing and reporting of results. For instance, in this report we observed that for each of the ESKAPE pathogens, the number of isolates tested for the various antimicrobial agents was different. We have not been able to report on colistin AST due to changed methodology by the CLSI and the European Committee on Antimicrobial Susceptibility Testing (EUCAST) in 2017. Data in this report represents a snapshot over only one year, thus long-term data are required for trend analysis.

\section{Conclusions}

K. pneumoniae and E. coli remained the commonest blood culture pathogens in the private sector for 2016. More than $50 \%$ of $K$. pneumoniae isolates and more than $15 \%$ of $E$. coli isolates were non-susceptible to the third- and fourth-generation cephalosporins, which is of concern. Carbapenem resistance among non-fermentative Gram-negative bacteria is alarming. Less than $50 \%$ of $A$. baumannii isolates were susceptible to carbapenems. From all $P$. aeruginosa isolates, $65 \%$ were susceptible to piperacillin/tazobactam. On a positive note, $75 \%$ of $E$. faecalis isolates were susceptible to penicillin/ampicillin with less than $1 \%$ showing non-susceptibility to the glycopeptides. However, as expected, only $8 \%$ of $E$. faecium isolates were susceptible to penicillin/ampicillin with less than $5 \%$ showing non-susceptibility to the glycopeptides. The prevalence of vancomycin resistance was higher among $E$. faecium isolates compared with E. faecalis isolates. Approximately one-quarter of all S. aureus bacteraemia was caused by MRSA. This report represents national data and it should be stressed that local and/or institutional level data should be utilised to help direct empiric treatment guidelines.

\section{Disclaimer}

Data are reported as received through the SIMU. No demographic, epidemiological, clinical or molecular data were available to distinguish between healthcare-associated and community-associated infections. CHARM is not responsible for testing or reporting results at patient level.

Acknowledgements - The authors wish to thank the following: Ms Sue Candy and her team at the SIMU for preparing the data; SASCM editorial committee (Prof O. Perovic, Dr W. Lowman, Prof. N. Govender, Dr C. Sriruttan, Dr K. Moodley, Dr C. Govind, Dr I. Zietsman, Dr B. Magazi, Dr R Kularatne, Dr M Maloba, Dr C. Bamford, Dr K. Swe Swe-Han and Dr Y. Mahabeer) for comments and suggestions.

Disclosure statement - No conflict of interest was reported by the authors.

\section{References}

1. Akova M. Epidemiology of antimicrobial resistance in bloodstream infections. Virulence. 2016;7(3):252-66.

2. World Health Organization [Internet]. Antimicrobial resistance: global report on surveillance, WHO 2014 [Updated June 2014; cited 20 October 2017]. Available from: http://apps.who.int/iris/bitstream/ 10665/112642/1/9789241564748_eng.pdf

3. Pogue JM, Kaye KS, Cohen DA, et al. Appropriate antimicrobial therapy in the era of multidrug-resistant human pathogens. Clin Microbiol Infect. 2015;21:302-12.

4. Performance Standards for Antimicrobial Susceptibility Testing. Clinical and Laboratory Standards Institute (CLSI), 2016; M 100-S26.

5. World Health Organization. Global antimicrobial resistance surveillance system: manual for early implementation. Geneva: World Health Organization; 2015. ISBN 9789241549400

Received: 10-01-2018 Accepted: 28-05-2018 